\title{
Recognition of notated melodies by possessors and nonpossessors of absolute pitch
}

\author{
KEN'ICHI MIYAZAKI \\ University of Niigata, Niigata, Japan \\ and \\ ANDRZEJ RAKOWSKI \\ Chopin Academy of Music, Warsaw, Poland
}

\begin{abstract}
Musically trained listeners compared a notated melody presented visually and a comparison melody presented auditorily, and judged whether they were exactly the same or not, with respect to relative pitch. Listeners who had absolute pitch showed the poorest performance for melodies transposed to different pitch levels from the notated melodies, whereas they exhibited the highest performance for untransposed melodies. By comparison, the performance of melody recognition by listeners who did not have absolute pitch was not influenced by the actual pitch level at which melodies were played. These results suggest that absolute-pitch listeners tend to rely on absolute pitch even in recognizing transposed melodies, for which the absolute-pitch strategy is not useful.
\end{abstract}

In a musical score (i.e., in the conventionally used musical notation system), the vertical position of the note head on the five-line staff represents pitch specified by frequency. However, musical scores also represent relative pitch information, with any arbitrary pitch as a reference. Given a reference pitch, musically literate people are able to realize a musical passage exactly as it is written in the score by using the relative-pitch sense. When no reference pitch is given and no support of accompanying instruments is available, listeners might be able to sing a melody accurately in relative pitch. By contrast, only people who have absolute pitch are able to sing the song with pitches exactly as notated, without recourse to reference pitch.

Absolute pitch (AP) is defined as an ability to name single pitches with no reference tone given and produce pitches corresponding to designated pitch names or musical note symbols. It has been commonly believed that AP is an extremely rare musical ability (Takeuchi \& Hulse, 1993; Ward, 1999). However, in spite of such remarkable aspects of AP, there is a possibility that AP possessors might have difficulty when they are required to sing notated melodies in the transposed key (i.e., at pitch levels different from the notation), if they realize the melody strictly on the basis of AP. This could raise important problems for musicians. For example, some musicians with AP have admitted that they feel extremely uncomfortable when they hear a familiar piece of music played with instruments tuned to a historical pitch stan-

Correspondence should be addressed to K. Miyazaki, Department of Psychology, Faculty of Humanities, University of Niigata, Niigata 950-2181, Japan (e-mail: miyazaki@ human.niigata-u.ac.jp). dard that is lower than the current one by nearly one semitone and complain that it is in the wrong key or that all the tones sound out of tune. Other musicians with AP have confessed that they are at a loss when they sing a song or play a singers' accompaniment in different keys from the actual score (see Moore, 1979).

It is obvious that musical pitch is intrinsically context dependent. Essential musical information, such as the melodic and harmonic characteristics of a musical passage, is conveyed by means of the pitch configuration in certain tonal contexts. The ability to deal with such pitch configurations is called relative pitch. By contrast, absolute pitch is not that essential to music; it may be relevant to music, at most, when musicians have to tune their instruments or voices to a standard reference pitch before beginning their performance.

Thus, previous investigations on music perception have mainly focused on the relational aspect of musical pitch, not on absolute pitch (see Krumhansl, 2000, for a recent review). For instance, typical experiments on melody recognition have used the delayed-comparison procedure, in which listeners hear a standard melody followed by a transposed, comparison melody and attempt to judge whether the two melodies are the same or different on the basis of their pitch relations (a sequence of musical intervals), regardless of absolute pitch (Bartlett \& Dowling, 1980; Cuddy \& Cohen, 1976; Dowling, 1978). In experiments in which tonal hierarchy has been investigated, a probe-tone technique has been used (see Krumhans1, 1990; Krumhansl \& Kessler, 1982, for a comprehensive review). Participants hear a probe tone preceded by context stimuli for establishing a particular tonal context and attempt to rate how stable the probe tone sounds in the tonal context. In this type of experi- 
ment, typically, the major or minor key context has been established on different tonal centers by providing a diatonic scale or a cadential sequence of chords at different pitch levels; as a consequence, the tonal profile obtained from this procedure will reflect the tonal hierarchy on the basis of pitch relations, again, independent of absolute pitch. Listeners with AP are purposefully excluded from the subject pools for these experiments, because it is suspected that AP listeners use peculiar strategies, providing results quite different from those who do not have AP. AP listeners have usually served as participants exclusively in experiments focusing on absolute-pitch processing. Therefore, it still remains unclear how AP listeners respond in recognizing melodies and when rating tonal hierarchies. Owing to the exclusion of AP listeners from experiments of perception of melodies and tonal hierarchy, these problems, until recently, have escaped investigation.

Tentatively, AP listeners are expected to perform better in recognizing tonal patterns, because AP might facilitate identification of musical pitch. This prediction is consistent with the view that AP is an important component of musical ability. However, when one considers that AP is musically irrelevant and is sometimes even incompatible with relative pitch, which is far more essential than AP in music, an alternative challenging hypothesis could be raised that AP listeners could have some disadvantages in certain tasks that require strategies incompatible with those dealing with absolute pitch.

In order to test this hypothesis, the ability to identify musical pitch relations (i.e., naming relative pitch) has been investigated in a series of experiments in which listeners having AP and those not having AP were studied (both groups had approximately the same degree of musical experience; see Miyazaki, 1993, 1995). The participants heard a pair of successive tones forming a differently sized melodic interval and attempted to name the pitch of the second tone relative to the first tone as a reference by using relative pitch names (i.e., sol-fa names in the movable-do system; e.g., re, $m i$, etc.). The results indicated that both AP listeners and non-AP listeners performed equally well when the musical intervals to be identified began with the $\mathrm{C}$ note; the listeners in both groups had been extensively trained in music. The AP listeners were able to identify musical intervals beginning with $\mathrm{C}$ by using both AP and relative pitch, whereas the non-AP listeners were able to identify the intervals by using only relative pitch. However, the AP listeners performed significantly worse when the musical intervals began with the F sharp or out-of-tune $\mathrm{E}$ flat note; as a consequence, the performance of the AP listeners was significantly worse than that of the non-AP listeners in these cases. These results have been interpreted to reflect an inevitable tendency for AP listeners to rely on AP in processing musical pitches. Presumably, the strategy of relying on AP was indeed effective for the AP listeners in identifying pitch relative to $\mathrm{C}$, because they were highly accustomed to naming relative pitch on the basis of the fixed-do system, naming $\mathrm{C}$ as $d o$; hence, the relativepitch naming task with $\mathrm{C}$ as the reference turned into an AP-naming task, a straightforward and easy task for the AP listeners. However, AP listeners had more difficulties in identifying relative pitch with the non-C reference, because here, they had to use the relative-pitch strategy, which is poorly used by them, or they would inappropriately stick to the AP strategy and try to transpose the pitch interval to be identified to their home position with the fixed $\mathrm{C}$ reference.

However, these results are not so straightforward as to be direct evidence for the disadvantage of AP, because the performance decline of the AP listeners could be accounted for, at least partially, by the naming system the AP listeners used for responding. The musical-interval identification task in previous experiments requires that participants name musical intervals on the basis of the sol$f a$ naming system. For non-AP listeners, the sol-fa labels are used as relative-pitch names, based on the movable-do system, in which any tone can be $d o$ according to the current tonal context. However, AP listeners typically use the sol-fa labels as the absolute-pitch names, based on the fixed-do system in which the C note is always $d o$, regardless of the key context. Hence, the AP listeners probably had a conflict in identifying relative pitch because of the labels they had to use in responding. This type of conflict could be regarded as an auditory analog to the Stroop interference effect typically observed in the visual domain (i.e., interference in naming the color in which a letter string is displayed when the letter string happens to be the name of an incongruent color word). This conflict is supposed to cause performance to decline when the relative-pitch name and absolute-pitch name are incongruent.

To further investigate the possible disadvantage of AP in identifying musical pitch in a tonal context, as has been suggested in previous experiments, in the present experiment, we employed a melody-comparison task, in which pitch labels were not used, in order to avoid the problems caused by the conflict between relative-pitch and absolute-pitch names. The purpose of the present experiment was to examine whether AP listeners have similar difficulties in recognizing transposed melodies as they do in recognizing transposed intervals. In each trial, a simple melody constructed for the experiment was presented visually in a form of musical notation; the participants then heard a comparison melody that was either at the same pitch level as the notated standard melody or was transposed to a different pitch level. The comparison melody was either the same or different from the standard melody with respect to relative pitch, irrespective of absolute-pitch levels; the same comparison melody was exactly the same as the standard, and the different comparison melody included one note shifted upward or downward by one or two semitones, with the constraint that the melodic contour did not change. A same-different dis- 
crimination task was used, in which the participants were to judge as fast as possible whether or not the comparison melody was exactly the same as the standard melody.

This procedure is different from typical procedures in the investigation of melody recognition. The procedure typically employed is that of delayed comparisons, in which both the standard and the comparison melodies are presented auditorily, and listeners attempt to judge whether the melodies are the same or different. In such experiments, the comparison melodies must be transposed to different pitch levels (different keys) from the standard in order to reliably examine melody recognition (e.g., Bartlett \& Dowling, 1980). If the comparison melody were to be presented at the same pitch level as that of the standard, the task, in effect, would just be a simple direct pitch-comparison task, which would be quite easy not only for AP listeners but also for non-AP listeners, owing to the use of short-term memory of absolute pitch, as long as the time delay is not too long. However, presentation of the standard melody in a notation, as in the present experiment, allows one to examine melody recognition for untransposed melodies, as well as for transposed melodies.

If AP listeners have a disadvantage in identifying musical pitch relations, as demonstrated in previous experiments, it is assumed that they will have the same difficulty in recognizing transposed melodies compared with untransposed melodies. In recognizing transposed melodies, listeners are required to process musical intervals and tonal characteristics in the context of a musical scale. If the AP listeners do show a disadvantage in the present task, it would serve as further evidence in support of the view that AP listeners have a strong tendency to use the AP strategy even when it is inadequate for recognizing pitch relations as musical intervals or melodies.

\section{METHOD}

\section{Stimulus}

In total, 120 standard melodies were composed, each of which had seven notes of equal duration. Half of the standard melodies were tonal, and the other half were atonal. The tonal melodies were constructed from diatonic scale tones of the major mode, strongly suggesting a major key, whereas the atonal melodies contained several nondiatonic tones, suggesting no definite key. The melodies differed in the number of reversals of melodic contour (the melodic shape complexity), from the simplest (one reversal) to the most complex (five reversals). The melodies always began with the central $\mathrm{C}$ and moved around in the range from lower $\mathrm{G}(\mathrm{G} 3)$ to central A (A4). The standard melody was displayed on a computer monitor in a musical notation format consisting of a sequence of crotchets placed on the musical staff with a treble clef.

Comparison melodies were presented auditorily at three different pitch levels. In some trials, the comparison melody was played at the same pitch level as the notation of the corresponding standard melody, beginning with the central $\mathrm{C}$ (the untransposed condition). In the other trials, the comparison melody was transposed four semitones lower than the notated standard, beginning with lower $\mathrm{G} \#$, or was six semitones higher, beginning with $\mathrm{F}^{\#}$ in the central octave (the transposed condition).
The combination of two levels of tonality (tonal vs. atonal) and three levels of transposition $(0,-4$, and 6 semitones) made for six experimental conditions, to which 20 melodies were assigned. Care was taken to equalize the melodic complexity among these conditions by having the number of reversals of pitch contour equally distributed (one reversal, 2; two reversals, 6; three reversals, 6; four reversals, 4; five reversals, 2). The melodic interval between adjacent tones in a melody ranged from 1 semitone to an octave, and the average of the pitch intervals in each melody was $2.17-6.33$ semitones. The distribution of the size of melodic intervals was roughly equivalent among the six experimental conditions (average, 3.323.66 semitones).

The comparison melody was either the same as or was different from the corresponding standard; the same comparison melody was identical to the notated standard with respect to pitch relations, whereas the different comparison melody had one of the central five notes changed one or two semitones upward or downward, with the restriction that the melodic contour was not violated by this change. For tonal melodies, the changed note shifted one step higher or lower on the diatonic scale, so that the amount of pitch change was dependent on the direction of the change and the position of the changed note in the diatonic scale. For melodies in the atonal conditions, the degree of pitch change was equalized in order to correspond to the tonal conditions. Of the 20 melodies in each experimental condition, 8 were the same and 12 were different (i.e., 4 melodies with a one-semitone pitch change, and 8 melodies with a two-semitone pitch change), and the temporal position of the changed note was equally distributed among all the conditions. In Figure 1, examples are shown of pairs of the tonal standard melody and its corresponding comparison melody in the different transposition conditions.

The comparison melodies were preceded by a two-chord sequence, which formed the conventional cadence (V7-I) of the major key, with the initial note of the comparison melody as the tonic. This chord sequence was introduced in order to enable the participants to anticipate the key of the tonal comparison melody or the beginning note of the atonal comparison melody. Each chord was $640 \mathrm{msec}$ in duration, and there was a 640-msec silent interval between the last chord and the first note of the comparison melody. Constituent tones of the comparison melody were about $280 \mathrm{msec}$ in duration, and the onset-to-onset time interval was $320 \mathrm{msec}$.

The chord sequence started at the same time as the visual display of the notated standard melody, which remained on until the participant responded. All the notes were presented in the piano timbre of a musical tone generator in moderate loudness.

\section{Apparatus}

The generation and presentation of the stimulus melodies and registration of the participants' responses were done with a computer (Macintosh PowerBook 170) and an Apple MIDI interface. Musical notation of the standard melody was displayed in the center of the computer screen in front of each participant. The sound of the comparison melody and the preceding chord sequence was generated from a tone-generator module (Roland, SC-88) and was presented to the participants through headphones binaurally. Each participant made a response by pressing one of designated keys on a musical keyboard (Korg). HyperCard software (Apple) and a programming language, HyperTalk, were used for controlling the experimental setup, and HyperMIDI external commands (EarLevel Engineering) were used for controlling the MIDI system (Miyazaki, 1998).

\section{Procedure}

Each experimental trial began with the presentation of a musical score of a standard melody in the center of the computer screen and a chord sequence followed by a comparison melody. The participants' task was to determine whether the comparison melody was 
(A) Tonal

Same Pair

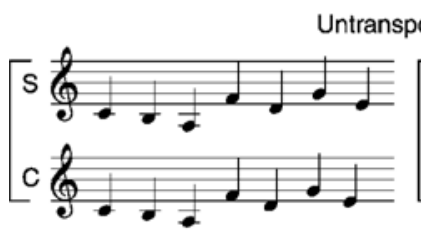

Transposed by 4 semitones downward
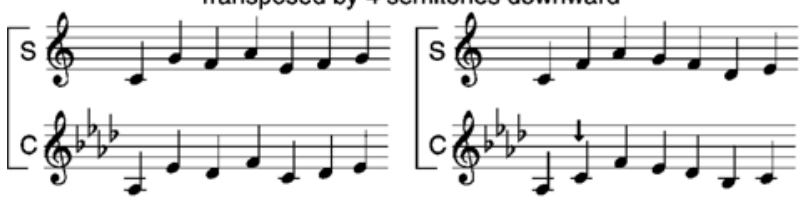

Transposed by 6 semitones upward
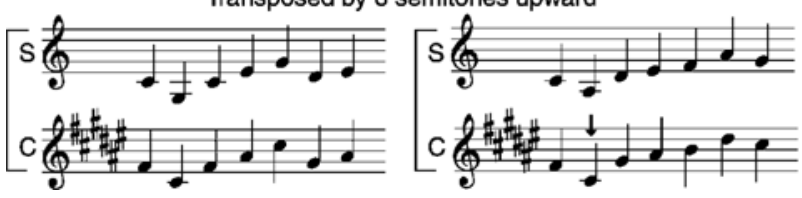

Different Pair

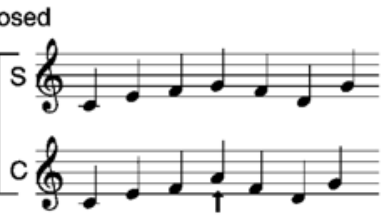

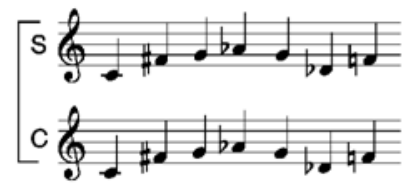
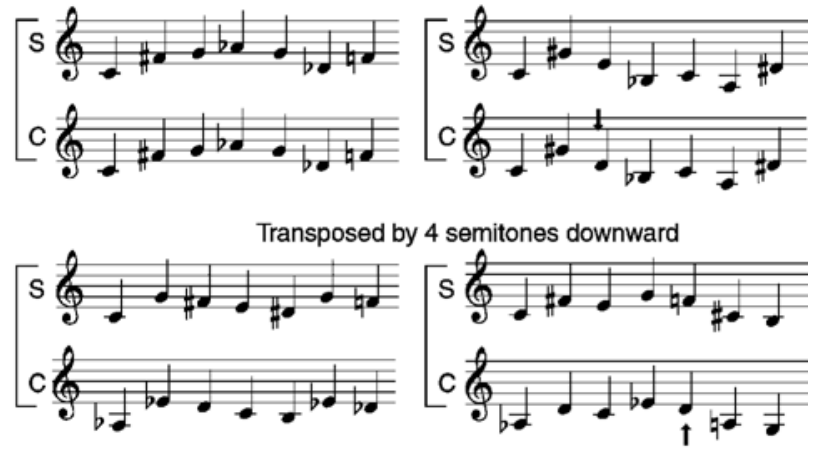

Transposed by 6 semitones upward
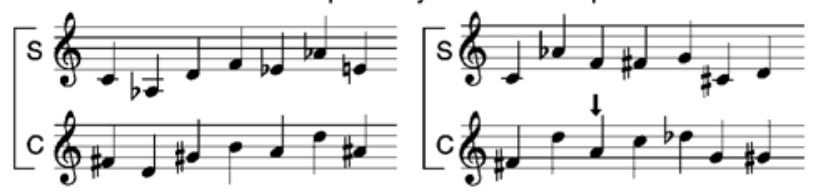

Figure 1. Examples of standard melodies $(S)$ and comparison melodies $(C)$ in the tonal (A) and atonal (B) conditions. Standard melodies were displayed visually in a format of musical notation always in the $\mathbf{C}$ major $\mathrm{key}$, and comparison melodies were presented auditorily either at the same pitch level (untransposed) as standard melodies or in different key (transposed by four semitones downward or six semitones upward). Note that the comparison melodies in the same pairs are exactly the same in relative pitch, and those in the different pairs include one tone shifted upward or downward by one or two semitones; the shifted tone is marked by an upward or downward arrow.

exactly the same as the notated standard or contained a modified pitch, in terms of relative pitch, without regard to the difference in absolute pitch. The participants were instructed to make a keypress as quickly as possible. Six consecutive white keys in the center of the keyboard were used for responding and were labeled "D3," "D2," "D1," "S1," "S2," and "S3," in this order, from left to right. The participants were instructed that they should press one of the three "D" keys when they judged the comparison melody to be different from the notated standard and that they should press one of the three "S" keys when they judged the heard melody to be the same as the standard. The number following " $D$ " or "S" represented the degree of confidence in judging; thus, the keys, as a whole, corresponded to a 6-point scale, with surely different $=$ D3 and surely the same $=\mathrm{S} 3$ as the extremes. The participants were allowed to make a response before the comparison melody came to an end if they detected the difference between the heard melody and the notated standard.

After a participant made a response, the window displaying the notated standard was replaced by a feedback window, which provided the participant with feedback concerning response correctness, and there was a response delay time after the onset of the last tone; in addition, there was a response history box in which a small open circle was added when a participant made a correct response (same response to the same trial or different response to the different trial), and a small closed circle was added when the response was incorrect (same response to the different trial or different response to the same trial). The participants were encouraged to get as many open circles as possible and to avoid closed circles, and to do so as quickly as possible. In this manner, it was expected that the participants' motivation would be kept high. Apparently, the participants found the task to be a sort of game. The experiment proceeded at the participants' pace; when the participants pressed any key after a response, the next trial began, after a delay of $1 \mathrm{sec}$.
There were three experimental sessions, each of which contained 120 trials ( 2 tonality conditions $\times 3$ transposition conditions, with 20 different melody sets each). Each session took approximately $25 \mathrm{~min}$; thus each participant was involved in 360 experimental trials. Actually, there were 20 uncounted warm-up trials prior to the experimental trials in each session. The experiment was carried out on 2 separate days; the first two sessions were given on the 1st day, and the last session on the 2 nd day. In each session, tonal and atonal conditions were arranged randomly, and the transposition conditions changed in every trial in an unpredictable manner. The three sessions had different orders of trials.

Before the melody recognition test on the 2nd day, the participants performed the absolute pitch test, in which 60 notes of the chromatic scale, based on equal temperament, were presented over the central five octaves. The AP test consisted of the same sampled piano tones as those used in the melody test. The fundamental frequencies ranged from $\mathrm{C}_{2}(65.4 \mathrm{~Hz})$ to $\mathrm{B}_{6}(1975.5 \mathrm{~Hz})$, with the pitch standard of $440 \mathrm{~Hz}$ as $\mathrm{A}_{4}$. The participants heard the test tones presented one by one in isolation and were to try to identify each pitch class and respond by pressing the corresponding key within a restricted octave range on a keyboard. Octave locations were not considered. The test tones were presented in a pseudorandom order, with the constraint that tones on consecutive trials were to differ by more than one octave and differ by more than three steps in the pitch-class circle in order to prevent the participants from relying on relative judgments made on the previously presented tones. There was a 3-sec intertrial interval between the response and the onset of the next tone. The same apparatus as in the main experiment was used.

\section{Participants}

The participants were 31 students recruited from the classes of solfège at the Department of Sound Engineering in the Chopin Academy of Music, Warsaw. According to the results of the AP test, 
9 AP listeners (correct responses: 60\%-100\%) and 18 non-AP listeners (less than 50\%) were identif ied. Four other participants failed to take the AP test because they were absent from the 2ndday session. There were 7 participants who had begun music training in early childhood (3-6 years old), all of whom were in the AP group. The participants were paid for their participation.

\section{RESULTS}

Of the 31 participants, 5 were excluded from the data analysis, 4 because they failed to complete all three sessions and the AP test and 1 non-AP listener because of trouble with the experimental setup.

In the present experiment, the participants were asked to decide whether the standard and comparison melodies were the same or different by using a 6-point confidence rating scale. However, most of the participants did not use the entire range of this scale, but in most cases, used the extreme categories (i.e., surely the same and surely different). Therefore, the six rating categories were collapsed into two response categories, same and different, from which percentages of correct responses were calculated. Figure 2 shows the averaged percentage of correct responses of the AP listeners (the AP group) and non-AP listeners as a function of the amount of transposition for the tonal and atonal melodies, separately. The AP group showed higher performance in the untransposed condition than in the transposed conditions, whereas the non-AP group performed almost the same, irrespective of whether the comparison melodies were transposed or not. As a consequence, performance of the AP listeners was better than that of the non-AP listeners for the untransposed melodies but was worse for the transposed melodies.
These observations were confirmed by a three-factor analysis of variance (ANOVA) of mixed design, with AP (AP vs. non-AP) as the between-subjects factor and transposition (no transposition, four-semitones downward, and six-semitones upward) and tonality (atonal vs. tonal) as the within-subjects factors; the Greenhouse-Geisser correction for inhomogeneity of variance was applied wherever appropriate. The main effect of tonality was significant $\left[F(1,24)=163.91, M S_{\mathrm{e}}=50.00, p<.001\right]$, indicating that, as expected, overall performance was reliably higher for tonal melodies than for atonal melodies. The main effect of transposition was significant $[F(2,48)=$ $\left.30.37, M S_{\mathrm{e}}=40.68, p<.001\right]$, and the interaction between transposition and AP was also significant $[F(2,48)=25.55$, $\left.M S_{\mathrm{e}}=40.68, p<.001\right]$. The interaction reflects the most important aspect of the results, in that the effect of transposition differed between the AP group and the non-AP group.

The cause of the interaction was investigated in additional analyses, which revealed that the simple effect of transposition was significant for the AP group $[F(2,48)=$ $\left.55.88, M S_{\mathrm{e}}=40.68, p<.001\right]$ but not for the non-AP group. Post hoc multiple comparisons (Tukey's HSD test) for the AP group showed that their performance with the untransposed melodies was reliably higher than with the two types of the transposed melodies $(p<.05)$, although there was no reliable difference between the two types of the transposed melodies. It is particularly interesting that the simple effect of AP was significant for the untransposed melodies $\left[F(1,72)=6.19, M S_{\mathrm{e}}=157.97\right.$, $p<.025]$ and for the upward transposed melodies $\left[F(1,72)=4.53, M S_{\mathrm{e}}=157.97, p<.05\right]$; it was marginally significant for the downward transposed melodies
(A) Tonal

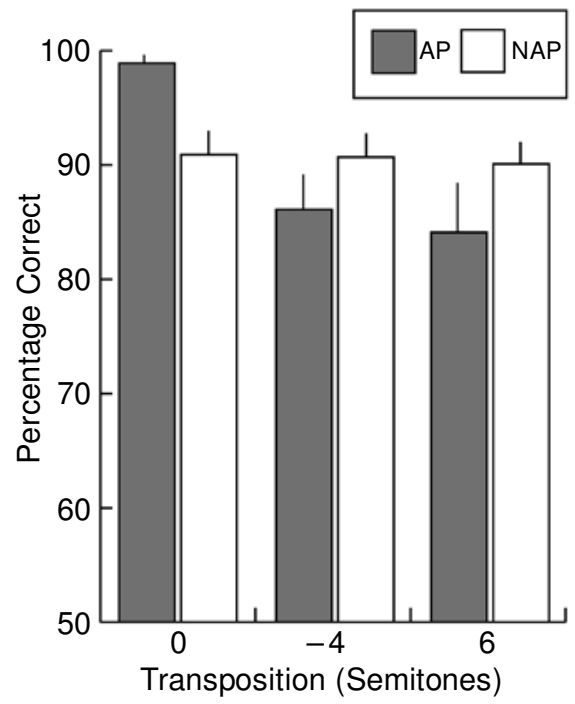

(B) Atonal

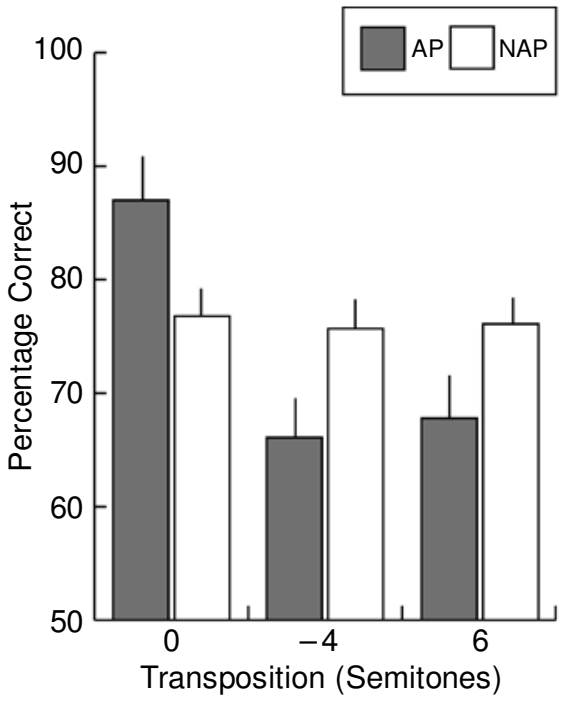

Figure 2. Averaged percentage correct responses as a function of the amount of transposition for the absolute-pitch listeners (AP) and the nonabsolute-pitch listeners (non-AP) (A) for tonal melodies and $(B)$ for atonal melodies. Bars show standard errors. 
(A) Tonal/AP

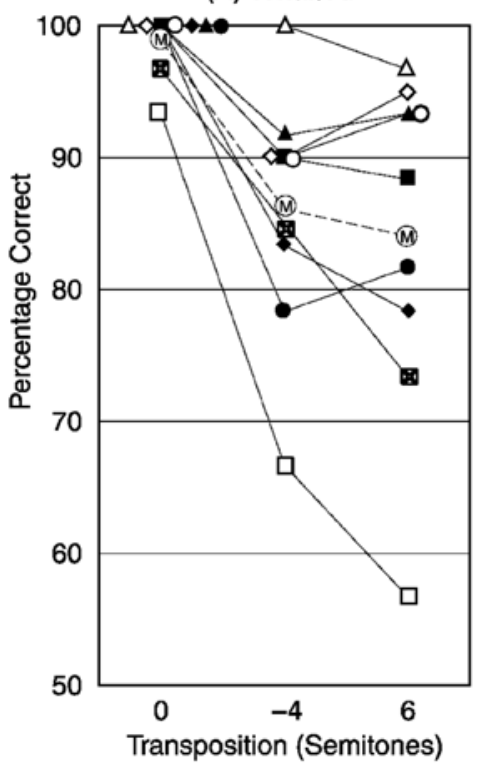

(C) Tonal/NAP

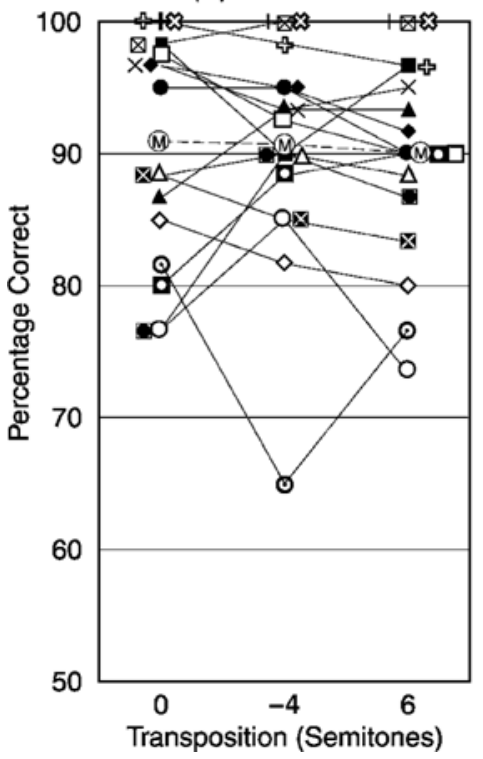

(B) Atonal/AP

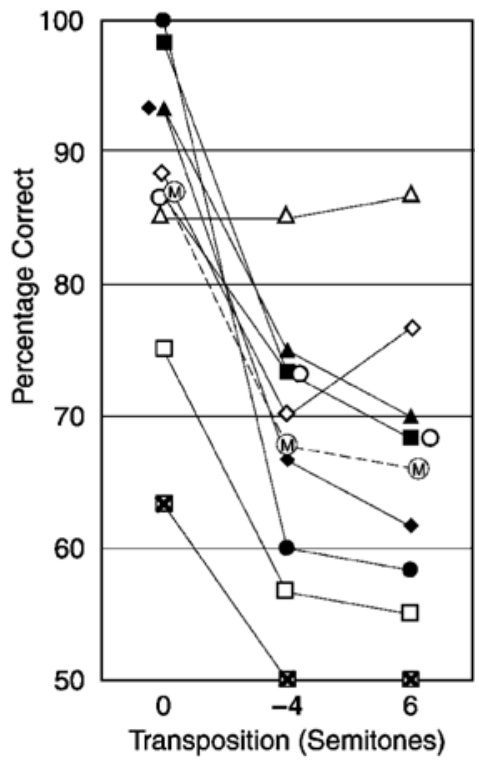

(D) Atonal/NAP

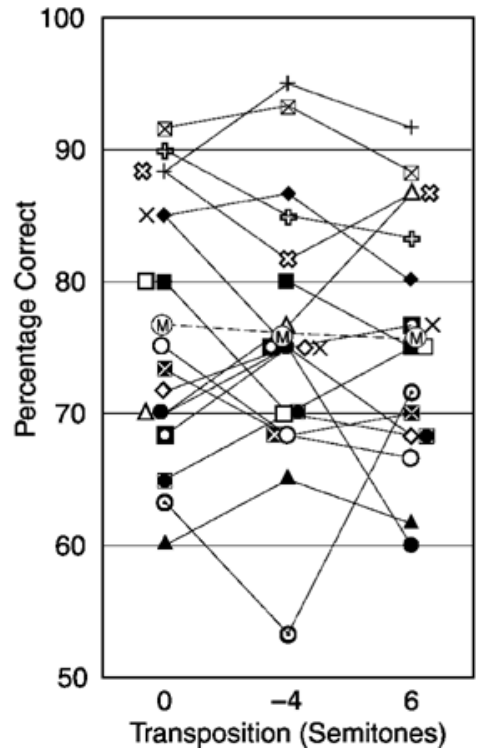

Figure 3. Percentage correct responses of individual listeners having absolute pitch (A and $B$ ) and those having no absolute pitch $(C$ and $D)$ for tonal melodies ( $A$ and $C$ ) and atonal melodies ( $B$ and $D$ ). The different connected symbols denote different listeners, and circled msec connected by a dashed line represent group averages.

$\left[F(1,72)=3.11, M S_{\mathrm{e}}=157.97, p<.1\right]$. This indicates that the AP group's performance was superior to that of the non-AP group, owing to the advantage of AP participants in recognizing untransposed melodies. However, the non-AP group had the advantage over the AP group in recognizing the transposed melodies.

There were large differences in performance among the participants. The percentages of correct responses for individual participants are presented in Figure 3. Dif- ferent symbols connected to the solid lines in each panel illustrate the performance of each individual for untransposed and transposed melodies. As can be seen, the performance levels are widely dispersed, particularly for the atonal melodies, for which the percentage correct ranges from near chance $(50 \%)$ to perfect. Most importantly, all the AP participants except one (represented by open triangles) showed a consistent decline in performance for the transposed melodies compared with the 
(A) Untransposed tonal melodies

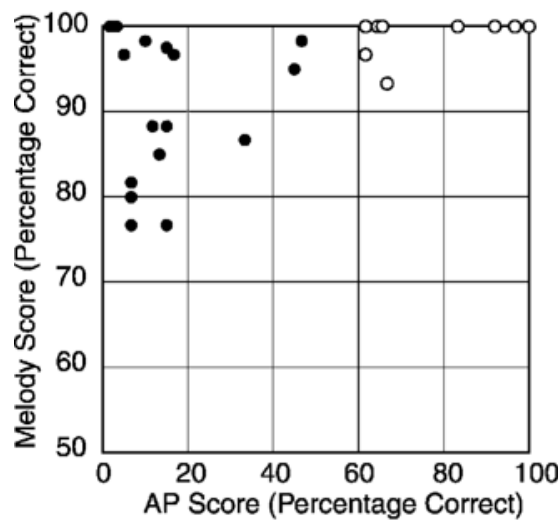

(C) Untransposed atonal melodies

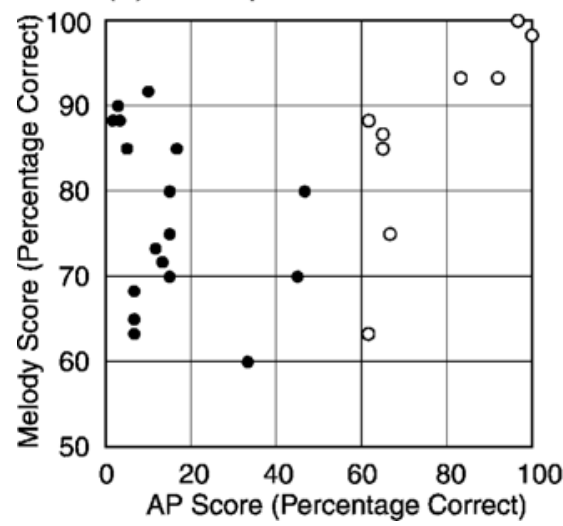

(B) Transposed tonal melodies

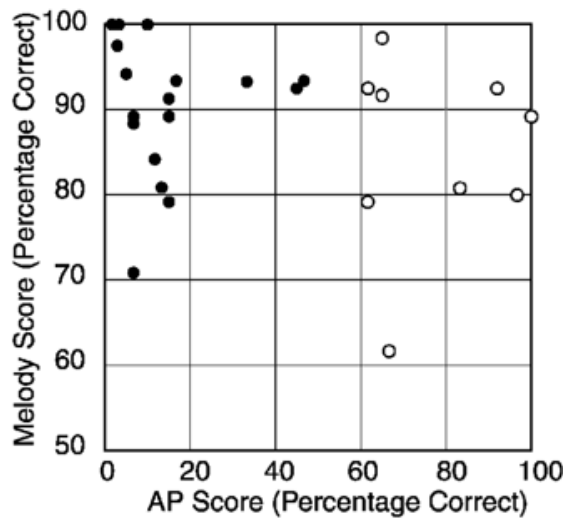

(D) Transposed atonal melodies

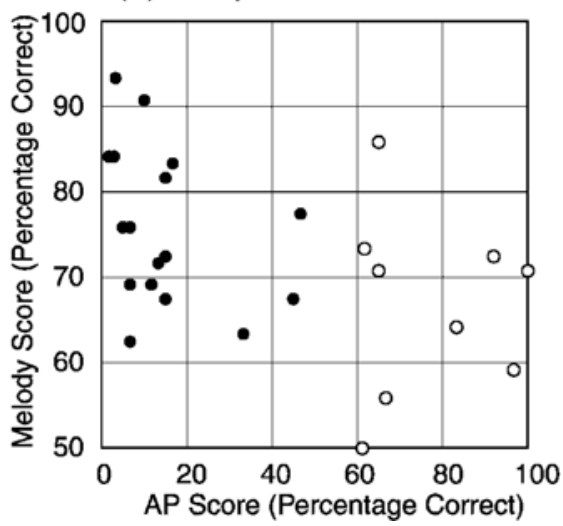

Figure 4. Scatterplot showing the correlation between the accuracy of melody recognition (abscissa) and absolute-pitch identification (ordinate). Open circles represent absolute-pitch listeners and closed circles represent nonabsolute-pitch listeners.

untransposed melodies. This trend is more pronounced for the atonal melodies (panel A) than for the tonal melodies (panel B). In contrast, most of the participants in the non-AP group showed no marked difference between transposed and untransposed melodies, although performance level varied widely among the participants.

Further analysis was carried out to examine the correlation between the accuracy of melody recognition (i.e., melody scores) and the accuracy of AP (i.e., AP scores). The results are represented in Figure 4 as scatterplots for the transposed and untransposed melodies, separately. Dots plotted in the scattergrams represent the individual participants, whose melody scores are plotted on the ordinate and whose AP scores are plotted on the abscissa. The scores for the untransposed melodies are plotted in the left-hand panels (A and C), and the scores for the transposed melodies (which were collapsed across transpositions of minus four and six semitones) are plotted in the right-hand panels (B and D). It is evident that, for the untransposed melodies, there was a significant positive correlation between the melody scores and the AP scores $(r=.48, p<.05$, for the tonal melodies; and $r=.44$, $p<.05$, for the atonal melodies). These positive corre- lations indicate that, in general, the more accurate the participants were in the AP test, the more accurate they were in recognizing untransposed melodies. By contrast, for the transposed melodies, negative correlations were found between the melody scores and the AP scores; the strength of the correlations was moderate for the atonal melodies $(r=-.45, p<.05)$ and negligible for the tonal melodies $(r=-.24$, n.s. $)$.

In order to examine further how the difference in performance between transposed and untransposed melodies was related to accuracy of AP, difference scores were calculated for individual participants by subtracting their melody scores (i.e., percentage correct) for the untransposed melodies from those for the transposed melodies. The difference scores were negatively related to the degree of accuracy of AP (i.e., the participants with more accurate AP tended to show larger decrements in recognizing transposed melodies relative to untransposed melodies; in contrast, the non-AP participants had smaller difference scores). The correlation between the difference scores and the AP scores was $r=-.62(p<.005)$ for tonal melodies, and $r=-.81(p<.005)$ for atonal melodies. 


\section{DISCUSSION}

The results of the present experiment show that transposition of melodies differentially influenced performance of AP listeners and non-AP listeners in melody recognition. The non-AP listeners exhibited equivalent performance, regardless of whether or not the comparison melodies were transposed from the notated standard melodies, indicating that they read relative-pitch information from the musical scores. In contrast, the AP listeners were strongly influenced by transposition; they exhibited the maximal level of performance for untransposed melodies, but showed the poorest performance for transposed melodies, indicating that they read absolutepitch information from the scores.

The equivalent performance of the non-AP listeners across transposition conditions is consistent with the notion in the Gestalt tradition in psychology that stresses the invariance of melodic identity under transposition as an important Gestalt property (i.e., transposed melodic patterns are perceptually, and musically, equivalent, in spite of the fact that all the tones of the melodies are changed in pitch by transposition, as long as the exact pitch intervals among tones are maintained). This idea was first formulated in 1890 by Ehrenfels $(1890,1937)$ in his papers on Gestalt qualities. The same idea was later addressed by Wertheimer (1938), who strongly emphasized that a melody-physically a sequence of definite individual tones-does not depend on activation of those particular tones, but has its own value that can best be described as a "Gestalt." The same melody can be recreated with the use of tones completely different from the previous ones (see also Deutsch, 1986; Krumhansl, 1990).

The performance of the AP listeners suggests costs and benefits of AP possession. The benefits of AP (i.e., the facilitated performance in untransposed-melody recognition) are not surprising because AP should offer direct benefits to AP listeners in comparing notated melodies with auditorily presented untransposed melodies. However, the cost of AP (i.e., the degraded performance for transposed melodies) is contrary to the commonly accepted view of AP being a very useful musical ability and so should deserve more attention.

Some caution might be exercised here, however, before claiming that AP has disadvantages. The criterion adopted here for defining the AP group was rather arbitrary. The participants in the present experiment were widely recruited from music students, and so only 3 listeners had accurate AP (more than $90 \%$ correct in the AP test). The listeners whose AP scores were higher than $60 \%$ correct were assigned to the AP group. It might be argued, therefore, that our AP group, defined according to such a liberal criterion, did not fairly represent AP possessors. It is true that there were a few inaccurate AP listeners who scored near chance for transposed atonal melodies, and these seemingly anomalous listeners reduced the average performance of the AP group for those melodies. Recalculation of the ANOVA was conducted with the anomalous AP listener who performed poorest being omitted. Significance of the main effects and the interactions did not change, but subsequent analyses showed that the simple effect of AP possession (the difference in performance of the AP and non-AP listeners) was reduced to a marginally significant level $(p<.1)$ for the transposed atonal melodies and to a nonsignificant level for the transposed tonal melodies. It could be argued that these follow-up analyses should not weaken the main results. In effect, there are varying degrees and qualitatively different types of AP. In one previous experiment, Miyazaki (1993) classified AP listeners as imprecise AP, partial AP (accurate only for white-key notes on the piano keyboard), and precise AP according to their AP scores, response patterns, and response speeds. He found that the partial and imprecise AP groups exhibited a more pronounced performance decrement in the relativepitch task than did the precise AP group. In that experiment, too, there were a few partial- and imprecise-AP listeners who performed near chance in identifying musical intervals beginning with notes other than C. Therefore, a few of the AP listeners of the present experiment who showed a similar decline in performance for the transposed melodies compared with the untransposed melodies are not anomalous but, rather, represent characteristics of some AP listeners in a somewhat exaggerated manner. Moreover, it is worth noting that the precise AP listeners showed a similar pattern of performance decline (see Figure 4), and there was a negative correlation between the AP scores and the percent correct for the transposed melodies.

It might seem counterintuitive to claim that AP is disadvantageous to music; superficially, it appears plausible that AP listeners are superior in recognizing tonal patterns, considering that absolute pitch facilitates all identification of musical pitch. In fact, there are a number of anecdotal reports of AP musicians who have achieved remarkable performance in many musical situations.

Indeed, AP is advantageous in music in two different ways. First, the advantage of naming pitch may be most useful in a music dictation task, in which one hears a musical passage and is required to write it on a staff. AP listeners are able to identify constituent tones of presented melodies and to place the corresponding notes on a musical staff. For extensively trained AP listeners, this process, based on the AP strategy, is supposed to be much easier than that based on relative pitch, as is evidenced by the remarks of one of the AP listeners that musical tones sound as if they are accompanied by their pitch labels. This might be very beneficial to AP listeners when given a dictation task including complicated tonal sequences that are difficult to perceive with relative pitch. It should be pointed out, however, that the improved performance achieved by the use of AP in a dictation test is irrelevant to music, in that performance does not reflect the ability to hear musical pitch relations, as assessed in the test, but simply reflects the AP ability instead. 
The second benefit of AP, the advantage of having pitch representations of a score, might also benefit musicians with AP in playing music and reading a score, particularly in sight reading. When given a score, AP musicians could easily create internal representations of the sequences by producing representations of individual notes and could then combine them into a melody representation. Thus, as in the dictation task, AP is highly useful for musicians who try to read music, particularly when they read a complicated score that includes sequences that are difficult to recognize, such as chromatic or dodecaphonic passages. Here again, however, it should be pointed out that the internal representation of the sequences formed with the aid of AP may not be a true musical entity that is constructed from the musical pitch relations that are directly read from the score, but instead may be a secondary construct assembled from the representations of individual tones.

Although the advantages of AP seem to be irrelevant to music, as is argued above, people with AP do exhibit higher performance in musical activities. However, the superiority AP listeners enjoy could come from the extensive musical training that they have received rather than from AP per se. Most of AP is a consequence of early musical training, and therefore, musicians with AP generally have had longer experience in musical training, beginning in earlier ages, than non-AP musicians (Miyazaki, 1988). Thus, AP possession and amount of musical training are usually confounded, making it difficult to differentiate between the contributions of these two factors to the higher performance of AP listeners in musical tasks. Therefore, it is unfair to compare AP listeners and non-AP listeners; a fair comparison would require the amount of musical experience to be equivalent for the two groups. When the difference in the amount of musical experience between the AP and non-AP groups is eliminated, the difference in performance observed between the groups could be taken to be reliable evidence for the pure advantage and/or possible disadvantage of AP.

In the present experiment, the participants were classified into the AP group and the non-AP group, solely on the basis of their accuracy on the AP test, without consideration of the amount of musical training they had received. Consequently, the participants in the AP group, independent of having absolute pitch, were musicians who possibly had been trained in music more extensively than those in the non-AP group. Because of this important difference, the AP listeners might naturally be expected to exhibit higher performance in general than would the non-AP listeners. Nevertheless, the results show that, contrary to what was predicted from the difference in musical experience, the performance of the AP group was lower than that of the non-AP group in transposed melody recognition. It could be possible that the difference would have been even larger if the AP group and the non-AP group had equal amounts of mu- sical experience. Therefore, the performance decrement of the AP group observed in transposed melody recognition is taken as even stronger evidence for the characteristic disadvantage of AP listeners rather than at face value.

When listeners try to recognize transposed melodies, AP does not work and even has adverse effects. If AP were under voluntary control, the AP listeners might have suppressed AP and switched to an alternative strategy based on relative pitch in the transposed condition. The finding that the AP listeners performed worse than the non-AP listeners in those conditions suggests that they have developed a strong tendency to rely on the AP strategy, and AP could be, for them, a sort of an involuntary, automatic listening strategy.

\section{REFERENCES}

Bartlett, J. C., \& Dowling, W. J. (1980). Recognition of transposed melodies: A key-distance effect in developmental perspective. Journal of Experimental Psychology: Human Perception \& Performance, 6, 501-515.

Cuddy, L. L., \& Cohen, A. J. (1976). Recognition of transposed melodic sequences. Quarterly Journal of Experimental Psychology, 28, 255-270.

Deutsch, D. (1986). Auditory pattern recognition. In K. R. Boff, L. Kaufman, \& J. P. Thomas (Eds.), Handbook of perception and human performance: Vol. 2. Cognitive processes and performance (chap. 32, pp. 1-49). New York: Wiley.

Dowling, W. J. (1978). Scale and contour: Two components of a theory of memory for melodies. Psychological Review, 85, 341-354.

EHRENFELS, C. vON (1890). Über “Gestaltqualitäten.” Vierteljahrschrift für wissenschaftliche Philosophie, 14, 249-292.

Ehrenfels, C. von (1937). Gestalt-qualities. Psychological Review, 44, 521-524.

Krumhansl, C. L. (1990). Cognitive foundations of musical pitch. New York: Oxford University Press.

Krumhansl, C. L. (2000). Rhythm and pitch in music cognition. Psychological Bulletin, 126, 159-179.

Krumhansl, C. L., \& Kessler, E. J. (1982). Tracing the dynamic changes in perceived tonal organization in a spatial representation of musical keys. Psychological Review, 89, 334-368.

MiYAZAKI, K. (1988). Musical pitch identification by absolute pitch possessors. Perception \& Psychophysics, 44, 501-512.

MrYAZAKI, K. (1993). Absolute pitch as an inability: Identification of musical intervals in a tonal context. Music Perception, 11, 55-72.

MiYAZAKI, K. (1995). Perception of relative pitch with different references: Some absolute-pitch listeners can't tell musical interval names. Perception \& Psychophysics, 57, 962-970.

MiYAZAKI, K. (1998). A system for experiments on music perception and cognition using Apple Macintosh and HyperCard. Journal of Music Perception \& Cognition, 4, 100-111. (In Japanese)

Moore, G. (1979). Am I too loud? Memoirs of an accompanist. London: Hamish Hamilton.

Takeuchi, A. H., \& Hulse, S. H. (1993). Absolute pitch. Psychological Bulletin, 113, 345-361.

WARD, W. D. (1999). Absolute pitch. In D. Deutsch (Ed.), The psychology of music (2nd ed., pp. 265-298). New York: Academic Press.

Wertheimer, M. (1938). Gestalt theory. In W. D. Ellis (Ed. and Trans1.), A source book of Gestalt psychology (pp. 1-11). London: Routledge \& Kegan Paul. (Original work published 1925)

(Manuscript received June 28, 2001; revision accepted for publication March 6, 2002.) 